\title{
Scientific Deconstruction of Two Folk Medicines
}

\section{Anjana Agarwal*}

Nutritionist and Aromatherapist, SNDT Women's University, Mumbai, India

*Corresponding author: Anjana Agarwal, Nutritionist and Aromatherapist, SNDT Women's University, Mumbai, India, Tel: +919958593488, E-mail: dranjanaagarwal@gmail.com

Received date: March 15, 2018; Accepted date: March 15, 2018; Published date: March 22, 2018

Copyright: @ 2018 Agarwal A. This is an open-access article distributed under the terms of the Creative Commons Attribution License, which permits unrestricted use, distribution, and reproduction in any medium, provided the original author and source are credited.

\section{Editorial}

Since time immemorial, people all around the world are managing their health issues with folk medicines using botanicals. World Health Organization (WHO) has also acknowledged it. Many ingredients used in folk medicines are readily available in the kitchen and surroundings. They have also been scriptured in Charaka samhita; Egyptian scrolls and Materia Medica which are compendium of innumerable formulations for numerous aliments and health conditions. In this piece of short communication, only two globally popular home remedies have been selected for scientific deconstruction. (1) Turmeric- milk (Golden milk) and (2) Lemon- honey water.

\section{Turmeric-milk}

Turmeric milk is an age old remedy and instant recall for quick healing of sprain, musculoskeletal diseases, immunity, tiredness and glowing skin. Turmeric milk is simply prepared by mixing turmeric and milk in varying proportions with or without sweetening agent like sugar or honey. In Indian medical systems, Ayurveda, "Turmeric" is used in innumerable health conditions. Turmeric is commonly used spice to impart golden yellow colour in Indian curries. Nutritionally, turmeric supply good amount of iron, manganese, niacin and vitamin B6. Each nutrient has biological function providing health benefits. It is highly valued for its bioflavonoids called curcuminoids such as curcumin, demethoxycurcumin and bisdemothoxycurcumin. Curcumin has globally shaken the pharmaceutical and nutraceutical world for its amazing health resolutions through its anti-inflammatory, anti-nociceptive, antimicrobial and antioxidant properties [1].

Anti-inflammatory property of curcumin is one of the most remarkable properties due to which it plays role in wound healing and injury. During injury inflammatory response it is a natural host defense. It triggers a cascade of biochemical events involving release of cytokines which alert the immune system, hormones and nutrients to reach the injured tissue for healing. Macrophages from immune system and prostaglandin (PGE2), hormone are released for healing. At the same time inflammation is associated with uncomfortable feeling of pain, redness, swelling and sometime fever. There are several proinflammatory markers such as proinflammatory cytokines, interleukin (IL 6), tumor necrosis factor (TNF- $\alpha$ ) and prostaglandin (PGE2), production of them is dependent upon the inflammatory enzymes like cyclooxygenase-2 (COX-2), 5-lipoxygenase (LO) enzymes. Anti-inflammatory compounds block these enzymes and also the biosynthesis of proinflammatory markers. During inflammation there is production of excessive free radicals and reactive oxygen species (ROS) which result in high oxidative stress level in the body, which itself is an indication of harmful effect in the body if not handled properly in time. Sumptuous supply of antioxidants can reduce the adverse impact of ROS. Curcumin and lactoferin from turmeric milk act as antioxidant and do the needful. Both compounds block the activity of cyclooxygenase-2 (COX-2) and 5-lipoxygenase (LO) enzymes and reduce the production of PGE2, cytokines and interleukins during injury and ameliorate the symptoms of injury. Curcumin molecule also blocks activation of pain receptors, i.e. transient receptor potential vanilloid 1 (TRPV1). Curcumin heals the wound by formation of fibroblast, collagen deposition, tissue remodeling and wound contraction. Body creates its own defense via antioxidant and one of them is glutathione. Curcumin induces biosynthesis of glutathione in the body. Glutathione itself inhibits production of inflammatory markers and also augments the production of catecholamines (collective name of norepinephrine, dopamine and serotonin) which demonstrate reduction in pain sensation and elevation of feeling of relaxation [2].

Milk is second crucial ingredient in turmeric milk. Milk is said to be an elixir in health and disease. It is a pool of nutrients and biologically active compounds such as lactoferin, lactoglobulins, immunoglobulins and lactoperoxidase which express antimicrobial, antibacterial, and anti-inflammatory, antioxidant immunomodulant, and hepatoprotective properties. Major proteins in milk consist of casein (insoluble) and whey (soluble). Whey protein is rich in branch chain amino acids (BCAA) and other amino acids which support biosynthesis of neurotransmitters, hormones and muscle fibres. Tyrosine supports release of dopamine and tryptophan synthesizes serotonin; Glutamine forms glutathione. Proline aids in production of collagen, an essential compound of tendons, cardiac muscles and joints and support healing and speedy recovery. Whey protein helps to remove toxins and ROS from body cells and improves mobility of tendons and joints. BCAA promotes healing of skin, muscle tissues and bones. Lactoferin, lysozymes and antibodies from the milk participate in wound healing process by formation of fibroblast within 2-3 days [3].

Milk contains a specific sugar i.e. lactose which improves absorption of calcium which in turn supports synthesis of collagen and improves contraction and relaxation of muscles. Lactose and short chain fatty acids (SCFA) from milk improve gut flora and immunity. Short chain fatty acids also modify the recruitment of circulating leukocytes to the inflammatory site and suppress the production of inflammatory cytokines. Conjugated linoleic acid (CLA) is another crucial fatty acid in milk possessing anti-inflammatory and antioxidant property thus plays role in suppressing inflammation process.

Curcumin is insoluble in water thus difficult to absorb in the body. It is advocated that curcumin if supplemented with piperine from black pepper improves its absorption in the body. Heat is also said to enhance bioavailability of curcumin. Black pepper itself is a therapeutic agent. Thus drinking hot turmeric milk during certain illness or injury is highly beneficial under the guidance of health professional. 


\section{Lemon honey water}

Morning drink of lemon honey water is a kick start of the day for health freaks. It boosts energy metabolism and aids in filtration of toxins from liver. People drink lemon honey water to elevate their work capacity and spirit and also to boost their immunity. Soothing power of honey, presence of vitamin $\mathrm{C}$ and bioflavonoids in lemon and hydrating capacity of water remarkably support for worldwide acceptance of lemon honey water. Let us unveil its other hidden secrets. First we will talk of lemon.

Centuries ago a surgeon of British Royal Navy saved lives of sailors with citrus fruits. Since lemons are highly valued for its richness in vitamin $\mathrm{C}$ and bioflavonoids. Vitamin $\mathrm{C}$ can provide protection from oxidative stress. It is an antioxidant and works in conjunction with vitamin $\mathrm{E}$ in cell membrane; it improves functioning of cells and reduces oxidative stress. Low vitamin $\mathrm{C}$ is associated with high BMI (Body Mass Index). This may support use of lemon water in weight reduction. A recent study on fasting with lemon honey water reveals reduction in body weight, BMI, body fat, high triglycerides. Fasting must have accentuated the results.

Bioflavonoids (or flavonoids) extend numerous health benefits and potentiate the function of vitamin C. Crucial bioflavonoid compounds in lemon are limonene, hesperidin, rutin and diosmin and each possesses specific role to support health. Delightful flavour and aroma of lemon is released by limonene found underneath its peel. Since limonene itself is a bitter compound thus over squeezing lemon makes the preparation bitter. Hesperidin helps to improve blood circulation and lung functions and also reduces inflammation thereby able to fight many diseases. Hesperidin with other compounds is now popularly used as functional food and in pharmaceutical and cosmetics preparations particularly under-eye dark circles. Similarly rutin also reduces vascular degeneration and swelling by improving the blood flow at vascular level thus found effective in leg cramps, varicose veins and eventually in cardiovascular diseases. Citric acid of lemons enhances the absorption of non-heme iron from plant sources there by reducing the iron deficiency anemia. Vitamin $\mathrm{C}$ supports collagen synthesis in connective tissues which helps to strengthen the joints and also make the skin firm, glowing and prevent wrinkle aggravation. Lemon water is thus considered an anti-aging drink. Lemons are acidic in taste but leave alkaline ash after utilization in the body thereby improve gut health. Lemon helps to alkalize the $\mathrm{pH}$ of the urine but does not affect the $\mathrm{pH}$ of the blood [4].

Water is a universal ingredient which in itself extends wide range of health benefits in the body such as transporting oxygen and nutrients to cells and removing metabolic wastes from there. It is necessary to maintain fluid and electrolyte balance, cellular communication and imbalance in them can cause havoc in the body. Dehydration is one of them. Drinking water is the first and best remedy to prevent dehydration. Often people do not realize that their physical tiredness and mental exhaustion is simply due to their dehydrated body. Using warm water is often calming and soothing to the gut thereby improves digestion and elimination process and also reduces risk of cramps and stiffness in muscles [5].
Miraculous power of honey is beyond a sweetening agent in this drink. Honey had always been on high pedestal in traditional medicine for centuries and now regaining attention in pharmacological avenues. Wide range of its chemical compounds demonstrates its virtues in healing the body and wounds. It is sweet and sticky in nature. Honey exhibits anti-inflammatory, antifungal, antiviral, antibacterial and antioxidant properties due to which it is useful in gastrointestinal, respiratory, cardiovascular, inflammatory and neoplastic diseases [6].

Honey owes its virtues to the presence of fructose, glucose, fructooligosaccharides, amino acids, vitamins, minerals, phenolics, peptides, organic acids, enzymes, and Maillard reaction products. Its functional flavonoids are apigenin, pinocembrin, kaempferol, quercetin, hesperedin ; phenolic acids like ellagic, caffeic, p-coumaric and ferulic acids and enzymes are catalase, superoxide dismutase, reduced glutathione and glucose oxidase. It is rich in minerals like potassium, calcium, magnesium, sodium, sulphur and phosphorus and trace elements like iron, copper, zinc and manganese. Amino acids, vitamins and trace elements have effect on regenerating tissues. Acidity of honey shows antibacterial activity due to which honey draws the moisture out from bacteria and dehydrates them. Glucose oxidase demonstrates antimicrobial property. It along with gluconic acid from its glucose improves calcium absorption. It is also anti-inflammatory and blocks the activity of COX -2 and synthesis of prostaglandins. It has shown reduction in C-reactive protein (CRP) levels in plasma, which is a good indicator of inflammation in the body. Honey has been well established to improve gut health and that is due to the presence of fructooligosaccharides and some organic acids which proliferates growth of bifidobacteria.

Besides these two folk remedies, other remedies having different compositions have also proven their potential in health and disease in practice and also in numerous research laboratories. However, they should also be used under supervision. Dose of the formula is critical to be effective. Excess is always risky. Certain ingredients may interact with medicines or supplements if taken together and may cause some unfavorable and risky reactions in the body. Cautiousness and consultation is always supportive.

\section{References}

1. Gupta C, Prakash D (2017) Therapeutic Potential of Milk Whey. Beverages 3: $31-45$.

2. Kapoor S (2012) Curcumin and Its Emerging Role in Pain Modulation and Pain Management. Korean J Pain 25: 202-203.

3. He Y, Yue Y, Zheng X, Zhang K, Chen S, et al. (2015) Curcumin, Inflammation, and Chronic Diseases: How Are They Linked? Molecules 20: 9183-9213.

4. Shetty P, Mooventhan, Nagendra HR (2016) Does short-term lemon honey juice fasting have effect on lipid profile and body composition in healthy individuals? J Ayurveda Integr Med 7: 11-13.

5. Eteraf-oskouei T, Najafi M (2013) Traditional and modern uses of natural honey in human diseases: a review. Iran J Basic Med Sci 16: 731-742.

6. Sears B, Ricordi C (2011) Anti-Inflammatory Nutrition as a Pharmacological Approach to Treat Obesity. J Obes 1-14. 